\title{
EL CONCEPTO DE EXPERIENCIA: DE KANT A HEGEL*
}

\author{
Gabriel Amengual ** \\ "La totalidad de la experiencia, \\ es decir, la existencia" \\ (Walter Benjamin) ${ }^{1}$
}

"De Kant a Hegel" es el enunciado del tema general, propuesto para este IV Simposio de Dialógica, la Sociedad Iberoamericana de Estudios de la Filosofía de Hegel, y nombra una de las grandes cumbres en la Historia de la Filosofía, de un relieve extraordinario en la Filosofía en general y en concreto en la cuestión que quisiera abordar en este escrito: la experiencia, un relieve quizás sólo comparable con el tramo de historia comprendido entre Platón y Aristóteles. En efecto, en esté arco de tiempo que va dè la filosofía crítica kantiana a la filosofía sistemática de Hegel se produce la transformación del concepto de experiencia moderno, el que podríamos denominar de la primera modernidad, dividida entre empiristas y racionalistas, y superando dicha división se abre a la profundización contemporánea que se va a dar en este concepto. El concepto de experiencia, tanto en Kant como en Hegel así como en el proceso de transformación que sufre con el paso de uno al otro, ofrece una gran

* Trabajo realizado en el marco del proyecto HUM 2004-02295, titulado "Ruptura de la tradición. Nihilismo y transformación del tiempo y la memoria en la formación de la conciencia actual", financiado por el Ministerio de Educación y Ciencia de España; leído en IV Simposio de Dialógica: "De Kant a Hegel”, Academia Nacional de Ciencias de Buenos Aires, 11-13 de octubre de 2006.

** Universidad de las Islas Baleares, Palma de Mallorca, España. gamengual@uib.es

${ }^{1}$ Benjamin, Walter, "Über das Programm der kommenden Philosophie" (1918), en: Gesammelte Schriften. Unter Mitwirkung von Th. W. Adomo und G. Scholem hrsg. v. Rolf Tiedermann und Hermann Schweppenhäuser, Suhrkamp, Frankfurt a M., 1991, vol. II/1, pp. 157-171, cita p. 171; vers. cast.: "Sobre el programa de la filosofía venidera" en: Iluminaciones IV: Para una critica de la violencia y otros ensayos. Introducción y selección de Eduardo Subirats, traducción de Roberto Blatt, Taurus, Madrid, 1991, pp. 75-84 (falta apéndice). 
complejidad. Aquí vamos a centrarnos en un solo aspecto, creo que nada marginal, a saber, la experiencia como constitutivo, como elemento o proceso constituyente del sujeto (tomado en su sentido más genérico), de forma que ella misma conforma el conjunto de condiciones de posibilidad para la apertura al mundo y la realización de nuevas experiencias, es más, es el conjunto que determina dicha apertura y la experiencia.

Con ello, en dicha transformación no sólo se produce la transformación del concepto moderno de experiencia, sino que se señala el campo en el que se va a debatir el concepto de experiencia en la filosofía contemporánea, aunque en este caso se traduzca en otros términos, tales como sí-mismo, subjetividad, existencia, identidad personal, identidad narrativa, etc. ${ }^{2}$.

\section{El concepto de experiencia en Kant}

En la modernidad la cuestión de la experiencia se plantea como cuestión gnoseológica, como problema de la teoría o crítica del conocimiento. En concreto la experiencia indica la referencia del conocimiento, a partir de la cual tiene que elaborarse, a la que ha de adecuarse, responder y corresponder, de la que tiene que dar razón o incluso la que ha de ser su contenido. "En la medida en que el entendimiento humano haya de ser fuente de algún tipo de certeza que vaya más allá de la mera autoconciencia, debe apuntar a algo que no sea él mismo"; ese algo, lo otro del entendimiento, "en relación con lo cual se puede plantear la cuestión de la certeza como objetividad que rebasa los límites del cogito, recibe el nombre de "experiencia"'3. Experiencia es, pues, de entrada ese algo, lo otro respecto del entendimiento, en relación con lo cual se puede y se debe plantear la cuestión de la certeza como objetividad. Así se presentan,

2 Como visión panorámica de la problemática actual puede verse Conill, Jesús, "Concepciones de la experiencia", en: Diálogo Filosófico núm. 41, 14 (1998/2) 148169; Nicolás, Juan A., "Experiencia de la crisis y crisis de la experiencia”, en: Diálogo Filosófico núm. 41, 14 (1998/2) 171-184; Freudiger, Jürg / Graeser, Andreas / Petrus, Klaus (Hg.), Der Begriff der Erfabrung in der Philosophie des 20. Jabrbunderts, Beck, München, 1996.

3 Vázquez Lobeiras, María Jesús, "Immanuel Kant: el giro copernicano como ontología de la experiencia”, en: Endoxa núm. 18 (2004) 69-93, cita p. 70. 
en términos generales, las filosofías de la experiencia o empiristas. Ese es fundamentalmente el planteamiento empirista, reaccionando contra el racionalista que supuestamente (o fácticamente, como en Descartes) quiere fundamentar el conocimiento en la autoconciencia. .

Estas filosofías de la experiencia habrian recibido su primera gran formulación en Locke, en cuya serie Kant vendría a ser el último eslabón. Ahora bien, "desde este punto de vista, la aportación del empirismo habría consistido fundamentalmente en convertir la experiencia en un grave problema y en haber conducido el enigma del conocimiento al callejón sin salida del escepticismo"4. En efecto, si la idea ha de corresponder a una sensación, si el juicio ha de corresponder siempre a un hecho, no hay duda que no sólo será imposible llegar a afirmaciones generales, sino que se hace imposible incluso la conexión de hechos y la formación de juicios. Tanto la conexión como la generalización parecen ser deudoras de una actividad que el entendimiento lleva a cabo desde y por sí mismo, espontáneamente.

En el marco del empirismo y el escepticismo, la experiencia se interpreta como el punto de partida del proceso cognoscitivo y como su contenido. Para Kant (1724-1804), en cambio, la experiencia aparece fundamentalmente como el resultado, como el producto de la actividad cognoscitiva, en la que necesariamente interviene como soporte todo el conjunto de condiciones interpuestas por la subjetividad humana. De todos modos, el concepto de experiencia en Kant es tan matizado y complejo, que se hace necesario hacer un esquemático recorrido por el amplio campo de su desarrollo, dejándonos guiar especialmente por la Crítica de la razón pura ${ }^{5}$.

${ }^{4}$ Vázquez Lobeiras, “Immanuel Kant: el giro copernicano...”, p. 70.

5 Como se sabe Hermann Cohen ha interpretado la $\mathrm{KrV}$ como una teoría de la experiencia (cfr. Kants Theorie der Erfabrung [1871, $3^{2}$ ed. modificada 1918], in: Werke, hrsg. v. Helmut Holzhey. vols. I-III Hildesheim: Olms 1987. Una interpretación seguida de la obra bajo este punto de vista también la ofrece Paton, H. J., Kant's Metaphysic of Experience. A commentary on the first balf of the Kritik der reinen Vernunft. 2 vols. London-New York 19361,19705.

Kant, Kritik der reinen Vernunft, en: Werke, hrsg. v. W. Weischedel, vol. III-IV,WBG, Darmstadt, 1975. (Vers. cast.: Crítica de la razón pura. Prólogo, trad., notas e índices de Pedro Ribas, Alfaguara, Madrid, 1978). Se cita siempre en el texto con la abreviatura $\mathrm{KrV}$ seguida de $\mathrm{A}$ y/o $\mathrm{B}$ y el correspondiente número de página de la $1^{2} \mathrm{y} / \mathrm{o} 2^{\mathrm{a}}$ edición. 


\subsection{La experiencia como conocimiento}

Como es sabido, Kant parte de una distinción del conocimiento entre empírico y puro, que se definen respectivamente por los conceptos de receptividad y espontaneidad. En este sentido puede afirmarse que Kant reconoce un conocimiento empírico, y en una primera aproximación a la experiencia, la definiría precisamente como conocimiento empírico. De todos modos el conocimiento empírico no ha de confundirse con la mera sensación, la cual sería fuente de conocimiento, lo que podríamos llamar el material previo o la materia del conocimiento, mientras que éste se distinguiría por ser una composición, cuya materia fundamental o primera serían las sensaciones, es decir, "lo que nosotros recibimos por medio de impresiones". Si la sensación o impresión sensitiva implica receptividad, pasividad por parte del sujeto, ser afectado por el objeto, el conocimiento empírico, en cambio, supone no sólo aceptación pasiva, sino una recepción activa, con todo lo que implica de acogida, de apuntar a la cosa, de intencionalidad.

Con ello creo que hemos indicado los dos significados más simples de experiencia en Kant: la sensación o impresión sensitiva y el conocimiento empírico ${ }^{6}$. De entrada hay que recordar que la experiencia así entendida no es todo el conocimiento. En efecto, si, según reza la primera afirmación de la $K r V$ (B 1): "No hay duda alguna de que todo nuestro conocimiento comienza con la experiencia", un poco más adelante aclara más añadiendo: "Pero aunque todo nuestro conocimiento empiece con la experiencia, no por eso procede todo él de la experiencia". Dicha afirmación parece indicar que la experiencia es el primer paso del conocimiento, la primera forma de conocimiento, pero no por ello todo el conocimiento procede de la experiencia. De manera que podríamos resumir el concepto kantiano de experiencia diciendo que, en sentido estricto, es "conocimiento de lo dado sensiblemente"7. Pero quizás más importante que este aspecto es el siguiente: la experiencia es el comienzo del conocimiento, no algo previo a él, de tal manera que el conocimiento empieza con la experiencia, puesto que la experiencia es conocimiento, el primer conocimiento. La experiencia

6 Cfr. Holzhey, Helmut, Kants Erfabrungsbegriff. Quellengeschicbtliche und bedeutungsanalytische Untersuchungen. Schwabe, Basel-Stuttgart, 1970, pp. 208-210.

${ }^{7}$ Holzhey, Kants Erfabrungsbegriff, p. 244. 
no es sólo el material previo al conocimiento, sino conocimiento, conocimiento propiamente dicho, elaborado.

Si la experiencia - en el sentido de lo recibido por la sensibilidad- no es todo el conocimiento, hay que suponer que se da otro elemento, independiente de la experiencia, que es el que aporta el entendimiento, sus conceptos puros o a priori, de tal manera que el conocimiento surge por la aplicación de los conceptos puros del entendimiento a las percepciones, al material diverso recibido por la sensibilidad. Es más, este otro elemento no es algo que posteriormente se aplique a la experiencia, sino que interviene ya en su misma constitución, por el mismo hecho de que ella es conocimiento ${ }^{8}$.

Con ello ya nos encontramos con un concepto de experiencia mucho más rico. Como afirma Kant: ' $\mathrm{La}$ experiencia es, sin ninguna duda, el primer producto surgido de nuestro entendimiento al elaborar éste la materia bruta de las impresiones sensibles" (KrV A 1). Y en una reflexión de los años en que Kant está elaborando la $K r V$ (1775) afirma: "Experiencia es una percepción comprendida" (Refl 4679)9 ${ }^{9}$. El entendimiento, por ser la facultad de los conceptos, por medio de ellos se convertirá en "el autor de la experiencia" (Urbeber der Erfabrung, KrV B 127) ${ }^{10}$.

La crítica que Kant dirige al empirismo es de no haber valorado el papel activo del entendimiento en la elaboración del conocimiento y por

${ }^{8}$ En efecto, la experiencia se basa en la representación de una síntesis necesaria y no puramente casual, rapsódica, de percepciones, cosa que ella por sí misma no puede darse, ello, en cambio, es propio no del juicio de experiencia (del juicio sintético a posteriori) sino de la síntesis en el juicio (del juicio sintético a priori). La primera síntesis es la "composición" (Zusammensetzung, compositio), la "síntesis de una diversidad cuyas partes no necesariamente se implican unas a otras"; mientras que la segunda es la "conexión" (Verbindung, nexus), "la síntesis de lo diverso, en la medida en que sus elementos se implican necesariamente unos a otros" (KrV B 201 nota). La necesaria síntesis en la experiencia no se basa en la experiencia.

9 Kant, Immanuel, Gesammelte Schriften, Akademie-Ausgabe, W. de Gruyter, Berlin, 1926 , vol. 17, p. 664. Sobre este período de Kant y la transformación del concepto de experiencia cfr. Vázquez Lobeiras, "Immanuel Kant: el giro copernicano..."; Hinske, Norbert, "Wandlungen en Kants Verständnis von Erfahrung", en: Experientia. X Colloquio Internationale. Roma 4-6 gennaio 2001, Leo S. Olschki, Firenze, 2002, pp. 425-434.

${ }^{10}$ Cfr. Holzhey, Kants Erfabrungsbegriff, pp. 217-219. 
tanto de la experiencia. Así afirma que Hume "tampoco advirtió que el mismo entendimiento podría quizá, a través de estos conceptos, ser el autor de la experiencia en la que se hallan sus objetos" ( $K r V$ B 127). En este sentido ya cabría interpretar que no hay nada previo al entendimiento, por lo que los neokantianos y Heidegger intentarán subrayar la unidad entre sensibilidad y entendimiento, entre la estética y la lógica, algo que Kant no pretendió separar sino al modo de análisis lógico o reconstrucción analítica de algo que se da conjuntamente.

Con ello ya tenemos los rasgos distintivos del planteamiento kantiano: papel activo del entendimiento $y$ la experiencia como conocimiento $y$, por tanto, como producto del entendimiento. Esta orientación de la investigación kantiana se hace explícita en la sentencia ya citada de la introducción a la $K r V$ B, con la que abre el libro: "No hay duda alguna de que todo nuestro conocimiento comienza con la experiencia" ( $K r V$ B 1). Esta es la parte que Kant concede al empirismo, pero si seguimos leyendo llegamos al segundo párrafo que comienza con esta sentencia complementaria y de alguna manera correctora: "Pero, aunque todo nuestro conocimiento empiece con la experiencia, no por eso procede todo él de la experiencia" ( $K r V$ B 1).

Hablar de estos dos rasgos puede dar a entender un dualismo, como una doble fuente de conocimiento, que, por lo menos en algunos sentidos, sería totalmente ajeno al pensamiento kantiano. Por ello hay que plantear la pregunta de cómo genera el entendimiento la relación, la referencia de los conceptos, de las representaciones con un objeto. $\mathrm{La}$ respuesta la encontramos en la misma definición de objeto que, según Kant, "es aquello con cuyo concepto se halla unificado lo diverso de una intuición dada" ( $\mathrm{KrV}$ B 137), es decir, que la acción del entendimiento consiste en unificar, en crear la síntesis de lo diverso de una intuición dada, de lo múltiple que ha ofrecido la sensibilidad. En ello radica la aportación del entendimiento, su actividad o espontaneidad.

Más radical e insuperable resulta dentro de este planteamiento otro dualismo de no menores consecuencias, a saber, la distinción entre fenómeno y cosa en sí. Dado que el conocimiento es siempre una elaboración del entendimiento, la cual a su vez se basa en lo percibido por la sensibilidad, puesto que "todas nuestras intuiciones no son más que una representación fenoménica” (KrV B 59), el conocimiento se ve limitado a lo 
que nos aparece, el conocimiento es incapaz de alcanzar la cosa en ella misma, lo cual resulta incluso algo contradictorio.

En la $\mathrm{KrV}$ Kant se propone estudiar las condiciones para el surgimiento de la experiencia ${ }^{11}$. El resultado más importante de su estudio es que la experiencia es siempre información ya elaborada, puesto que lo que experimentamos depende de manera decisiva de nuestras propias capacidades de intuir (la sensibilidad) y pensar (el entendimiento). Sus consideraciones sobre la interacción de estas dos capacidades del sujeto han encontrado su expresión más representativa en aquella afirmación también muy repetida:

\begin{abstract}
"Sin sensibilidad ningún objeto nos sería dado $\mathrm{y}$, sin entendimiento, ninguno sería pensado. Los pensamientos sin contenido son vacíos; las intuiciones sin conceptos son ciegas. Por ello es tan necesario hacer sensibles los conceptos (es decir, añadirles el objeto en la intuición) como hacer inteligibles las intuiciones (es decir, someterlas a conceptos). Las dos facultades o capacidades no pueden intercambiar sus funciones. $\mathrm{Ni}$ el entendimiento puede intuir nada, ni los sentidos pueden pensar nada. El conocimiento únicamente puede surgir de la unión de ambos" ( $K r V$ B 75).
\end{abstract}

Consecuentemente en su filosofía trascendental Kant se propone la tarea de analizar más detalladamente estas facultades y mostrarlas como las condiciones de la experiencia, previas a toda experiencia; esto es precisamente lo que significa trascendental. Ahora habría que ver si la experiencia juega algún papel en este equipamiento trascendental, a priori, dado por las mismas estructuras de la subjetividad; habría que preguntar si la experiencia tenida revierte configurando las condiciones de posibilidad de nuevas experiencias.

${ }^{11}$ Kirchner, Katrin Jona, 'Die Funktion existentieller Erfahrungen des Selbst- und Fremdbezug in Franz Rosenzweigs Grundlegung der menschlichen Personalität und der intersubjektiven Beziehung", en: Ricken, Friedo ( $\mathrm{Hg}$.), Religiöse Erfabrung. Ein interdisziplinärer Klärungsversuch, Kohlhammer, Stuttgart, 2004, pp. 165-177, ref. pp. $165 s$. 


\section{2. ¿La experiencia como trascendental?}

La unificación de lo múltiple de las sensaciones no es algo posterior a la experiencia, sino lo que la constituye, constituyendo al mismo tiempo el conocimiento. Así Kant puede definir la experiencia como "una conexión sintética entre las intuiciones"12 y como "un conocimiento obtenido por medio de percepciones enlazadas"13. Lo cual confirma el carácter de conocimiento que es propio de la experiencia y a la vez explica la necesidad que la experiencia tiene del otro elemento.

Ahora hay que dar un paso más en la consideración del conocimiento. En efecto, para conocer se requiere no sólo la referencia de los conceptos puros del entendimiento a la multiplicidad de la sensación, sino también se requiere integrar lo percibido dentro de la unidad de lo pensado. Tomar conocimiento de algo significa siempre integrarlo dentro del conjunto de nuestro conocimiento, lo cual sólo es posible si se pone bajo el conjunto de las categorías. Llevar un objeto a concepto significa ordenarlo en el conjunto de la experiencia, dentro de la cual el objeto adquiere su lugar mediante su fijación conceptual. No cabe el aislamiento del concepto del objeto dentro de los principios que regulan la experiencia.

Siendo esto así, hay que preguntarse por cuál es la síntesis suprema, originaria, que precede a las síntesis singulares y a los complejos de representaciones. Dicha síntesis suprema no es otra que "El yo pienso tiene que poder acompañar todas mis representaciones" ( $K r V$ B 132). El yo kantiano, del que aquí se trata como el origen de la unidad sintética de la apercepción, es uno con la realización de la síntesis originaria, es esta unidad misma. Se da una perfecta identidad de yo y pensar. De la actividad de un yo no tenemos ninguna percepción, no es una consecuencia del acto mental, sino que es este mismo acto mental. El yo no es más que el sujeto formal de una operación unificadora del entendimiento. El "yo pienso" es el golpe que de una percepción hace una experiencia. Apercepción es la expresión técnica para este procedimiento. En la aplicación de este término, tomado de Leibniz, Kant no exige, a diferencia de Leibniz, el carácter consciente de las representaciones. Pero sí hace valer que todas las representaciones deben estar bajo las condiciones de la unidad de la autoconciencia. Es más, el

12 "Eine synthetische Verbindung der Anschauungen", $K r V$ B 12.

13 "Erkenntnis durch verknüpfte Wahrnehmungen", KrV B 161. 
ámbito de la experiencia posible coincide con el ámbito de las representaciones conscientes, por tanto con el ámbito de la conciencia posible. La unidad de las categorías viene garantizada por la unidad de todas las acciones mentales. El yo no es más que pensar, pero no es que sólo piense, sino que es el punto de referencia al que está orientada la unidad sintética de la apercepción, es el principio unificador del conocimiento mismo. De esta manera a priori podemos estar seguros de que los objetos de la experiencia sin excepción corresponden a los conceptos del entendimiento y así a los principios del entendimiento puro, porque los objetos sólo pueden ser construidos por nosotros a partir de la multiplicidad de las intuiciones con la ayuda de las categorías. Las categorías son condiciones de la posibilidad de la experiencia.

De esta manera nos encontramos con el papel decisivo de lo que en términos generales cabe llamar la conciencia. Que una representación empírica sea consciente es lo que la distingue de una intuición empírica. Gracias a la pertenencia a la conciencia la percepción es, por una parte, toma de conocimiento de algo objetivo, y, por otra, permite establecer la conexión con otras percepciones pudiendo llegar a la unidad de la experiencia.

La conciencia, pues, aparece bajo una doble figura. Por una parte, la conciencia empírica, la que percibe, la que se da en cada percepción que por ella se convierte en experiencia y en conocimiento; y por otra, la conciencia que permite la unificación de las percepciones, la conciencia trascendental o apercepción. En efecto, que la conciencia empírica de la percepción esté fundada en una conciencia originaria y que por tanto la síntesis de la aprehensión "ha de conformarse necesariamente a la síntesis de la apercepción" ( $K r V$ B 162 nota), constituye el pensamiento fundamental de la deducción trascendental de los conceptos puros del entendimiento. Lo dado en cada sensación no sería cognoscible como real, si de entrada, anticipadamente, no se le concibiera bajo la categoría de realidad. Este concepto de lo real, "no significa más que la síntesis realizada en una conciencia empírica en general" ( $K r V$ A 175/B 217), es decir, aquella representación a priori, que hace que la conciencia sea empírica, es decir, conciencia de algo que ella misma no se da de sí misma ${ }^{14}$.

${ }^{14}$ Holzhey, Kants Erfabrungsbegriff, pp. 228s. 
La conciencia empírica se halla necesariamente dispersa en una multiplicidad de representaciones, que ella acompaña, pero, a su vez, dichas representaciones son unificadas en su referencia a la conciencia originaria. La conciencia originaria es la conciencia del sí-mismo idéntico: la pura u originaria apercepción, bajo cuya unidad sintética, trascendental, están todas las representaciones propias del yo y así son conectadas. La unidad trascendental de la autoconciencia es la condición suprema de toda síntesis de representaciones, también de aquella unidad sintética que hace posible el uso del entendimiento en la experiencia y por tanto hace posible a ésta misma. La experiencia trascendental del sí-mismo es el a priori propio de la experiencia en general ${ }^{15}$.

No es ahora el momento de tratar de la experiencia del sí mismo. Simplemente cabe señalar que con ello Kant viene a recepcionar el "cogitans sum" de Descartes para -con la distinción entre apercepción trascendental y empírica- guardarse de toda objetivación como "res cogitans". Ahora bien, las consideraciones de Kant desembocan en la caracterización de la apercepción original como una conciencia trascendental (en contraposición a la empírica). Y seguramente consciente de los malentendidos de su propia exposición en $K r V$ B, en un apunte de 1788-1790 señala que la conciencia que pone en marcha una experiencia o también en general el pensar, es una conciencia trascendental, no la experiencia ${ }^{16}$. Para la conciencia es constitutivo el ser enseñada por los sentidos, lo cual no se da cuando soy

${ }^{15}$ Holzhey, Kants Erfabrungsbegriff, pp. 233. Como sucintamente afirma G. Agamben: "El viejo sujeto de la experiencia vuelve a presentarse aquí autónomamente como yo empírico, que es 'en sí disperso y sin relación con la identidad del sujeto', y como tal es incapaz de fundar un conocimiento verdadero. A su lado, como condición de todo conocimiento, está el yo pienso, la conciencia trascendental, es decir, la unidad sintética originaria de la conciencia, 'sólo gracias a la cual puedo atribuir a un idéntico yo mismo la multiplicidad de mis representaciones' y sin la cual la experiencia ya no sería conocimiento, sino solamente 'una rapsodia de percepciones"'. Giorgio Agamben, Infancia e bistoria. Destrucción de la experiencia y origen de la bistoria, Hidalgo, Buenos Aires, ${ }^{32004, ~ p . ~} 38$.

16 Refl. 5661. Se trata de un apunte, titulado "Beantwortung der Frage, ist es eine Erfahrung, dass wir denken?”, en: Kant, Gesammelte Schriften, Akademie-Ausgabe, vol. 18, pp. 318-320. 
simplemente consciente de que pienso y no de lo pensado. La primera es la conciencia trascendental, la segunda la empírica.

Con ello queda claro que lo que hace posible la experiencia no pertenece a ella, es anterior e independiente de ella. Las disposiciones o estructuras del sujeto que lo hacen capaz de experiencia y "autor de la experiencia" ( $K r V$ B 127), son estructuras a priori, que se dan en todo sujeto, vienen dadas por virtud de su propia subjetividad, independientemente de toda experiencia. El principio unificador de la experiencia y, por tanto, constitutivo de la experiencia en su conjunto, es algo anterior e independiente de la experiencia: es el sujeto trascendental, con sus estructuras transcendentales. De este modo "el planteamiento más riguroso del problema de la experiencia termina fundando su posibilidad a través de la posición de lo inexperimentable"17.

Con este planteamiento tenemos ya el dualismo entre lo recibido a posteriori y lo dado a priori, entre lo empírico y lo trascendental, entre lo experimentable y lo inexperimentable, entre lo múltiple de lo sensible y lo uno y unificador del sujeto. El sujeto resulta ser lo que no sólo no es configurado por la experiencia, sino que es ya por sí mismo anterior a toda experiencia, aunque sea el que la haga posible y la configure. La experiencia en ningún momento es considerada como un conjunto de condiciones que hacen posible la experiencia, nuevas experiencias, que configura las nuevas experiencias, aunque conocer, hacer experiencia, consista en integrar lo nuevo dentro del conjunto de lo conocido. En ningún momento se da a la experiencia el rango de trascendental, porque el sujeto está dado ya desde siempre y por sí mismo.

\subsection{Límites}

A partir de ahí ya se pueden señalar dos limitaciones a este planteamiento de la cuestión de la experiencia:

La primera tiene que ver con el modo cómo la experiencia llega al sujeto. Kant lo determina solämente a partir de las disposiciones propias del sujeto, y del sujeto en general, por tanto ahí no ha lugar a intereses, afectos, fines que guían, etc. El sujeto recibe o accede a la experiencia en virtud de

${ }_{17}$ Agamben, Infancia e bistoria, p. 39. 
las disposiciones o estructuras trascendentales que le son características como sujeto.

La segunda limitación viene a complementar la primera, en el sentido que Kant intenta describir las estructuras del sujeto independientemente de su persona o historia personal. Se trata de un sujeto sin historia y $\sin$ sociedad. Los contenidos de experiencia que adquiere cada individuo -que objetivamente pueden considerarse como contingentes- y que pueden ser decisivos en su vida, no juegan ningún papel. El hombre sólo aparece como sujeto racional abstracto. En este sentido es significativa la limitación sistemática sobre las capacidades, o a una teoría trascendental del alma, sin siquiera mencionar algo tan importante como los estados de ánimo. El sujeto del que se trata es el yo, la representación 'yo', “que es simple y, por sí misma, completamente vacía de contenido. Ni siquiera podemos decir que esta representación sea un concepto, sino la mera conciencia que acompaña cualquier concepto. Por medio de este yo, o él, o ello (la cosa) que piensa, no se representa más que un sujeto trascendental de los pensamientos $=\mathbf{x}$, que sólo es conocido a través de los pensamientos que constituyen sus predicados y del que nunca podemos tener el mínimo concepto por separado" (KrV B 404).

\section{El concepto de experiencia en Hegel}

Hegel (1770-1831) detectó esta insuficiencia en el planteamiento kantiano ${ }^{18}$. Kant ha descrito las condiciones de posibilidad trascendentales, es decir, dadas en la estructura psíquica del sujeto, pero su insuficiencia fundamentalmente consiste en que no se describe lo que pasa en el sujeto cuando hace una experiencia. Los análisis kantianos de las formas de la intuición en la facultad de la sensibilidad, de las categorías en el entendimiento y de la capacidad de abstracción de la razón no dicen nada de lo que sucede en el sujeto cuando hace una experiencia.

\subsection{La Fenomenología como ciencia de la experiencia}

Esta complementación o corrección de la teoría del conocimiento kantiana la podemos ver especialmente en la Fenomenología del espiritu de

${ }^{18}$ Kirchner, "Die Funktion existentieller Erfahrungen...”, p. 166. 
180719. En efecto, esta obra se inscribe dentro del contexto de los inicios del idealismo, a finales del siglo XVIII y principios del XIX, cuando se trabaja sobre el trasfondo de la idea de una historia de la autoconciencia ${ }^{20}$. En este sentido las otras dos obras anteriores de referencia son Grundlage der gesamten Wissenschaftslebre (1794-95) de Fichte y System des transzendentalen Idealismus (1800) de Schelling. También se han señalado paralelismos con las "novelas de formación" o de aprendizaje (Bildungsromane), de las que Wilhelm Meisters Lebrejabre (1795-96) de Goethe habría establecido las pautas arquetípicas ${ }^{21}$. Pero dejamos el vasto campo de la literatura para concentrarnos, y de manera esquemática, en el no menos vasto de la filosofía.

Fichte propone una parte de su Doctrina de la Ciencia como "historia pragmática del espíritu humano"22, Ahora bien, la historia del espíritu humano es sólo una ficción necesaria por cuyo medio debe exponerse el punto de vista de la conciencia no filosófica en sus consecuencias. En cambio, el Sistema del idealismo trascendental de Schelling es desde su inicio una "historia de la autoconciencia"23, no meramente ficticia, sino que integra también la historia real.

19 Hegel, Pbänomenologie des Geistes. Hrsg. v. Johannes Hoffmeister, Meiner, Hamburgo, 1952. (Vers. cast. de Wenceslao Roces y Ricardo Guerra: Fenomenología del espiritu, FCE, México, 1971). Se cita con la sigla $P b d G$ y el número de página del texto alemán y del castellano separados con una /).

20 Bonsiepen, Wolfgang, "Phänomenologie des Geistes", en: Pöggeler, Otto, (Hrsg.), Hegel. Einfuibrung in seine Philosopbie, Alber, Freiburg/München, 1977, pp. 5974 , ref. p. 64.

${ }^{21}$ Cfr. Hyppolite, Jean, Génesis y estructura de la Fenomenología del espiritu' de Hegel, (1946), Península, Barcelona, 1973, pp. 13s.; en este mismo sentido subraya que el tema de la Fenomenología no es la historia ni una filosofía de la historia, sino el proceso de formación de la conciencia, pp. 28ss., 34ss.

${ }_{22}$ Fichte, Johann Gottlieb, Grundlage der gesamten Wissenscbaftslebre $\left(1794,1798^{2}\right)$, en: Werke, ed. por I.H. Fichte, Berlín 1845-1846. Reimpresión W. de Gruyter, Berlin, 1971, vol. I, pp. 27-328, cita p. 222.

${ }^{23}$ Schelling Friedrich Wilhelm Joseph, System des transzendentalen Idealismus. Mit einer Einleitung von Walter Schulz, Meiner, Hamburgo, 1957; vers. cast.: Sistema del idealismo trascendental. Traducción, prólogo y notas de Jacinto Rivera de Rosales y Virginia López Domínguez, Anthropos, Barcelona, 1988. En realidad la propuesta de "Ta historia de la autoconciencia" Schelling la concibe ya en su estudio de Fichte, que cristaliza en el artículo de 1797, publicado después como libro en 1809 bajo el título Abbandlungen zur 
El proyecto de Hegel presenta elementos en común con estos proyectos y elementos diferenciadores de uno y otro proyecto. Con Fichte tiene en común que tampoco Hegel permite a la reflexión filosófica que expone la historia pragmática del espíritu humano que legisle acerca de dicha historia, sino que sólo le compete el mirar, el observar atentamente (Zusehen). Según Fichte, nosotros los que reflexionamos filosóficamente, nos comportamos como "historiadores [Gescbichtsscbreiber] pragmáticos", que únicamente siguen la reflexión natural del espíritu. Con la idea de la "historia de la autoconciencia" de Schelling, en cambio, Hegel tiene en común que la historia universal de la autoconciencia no es sólo ficticia, sino que se integra dentro de la historia real.

En cambio, Hegel se diferencia de ambos por la conexión inmanente, necesaria que establece entre las diferentes figuras de la conciencia, de manera que, dentro de una dialéctica de diferentes figuras de conciencia, quiere deducir la estructura de la autoconciencia de la estructura de la conciencia, por la cual viene a sustituir la "historia pragmática del espíritu humano" de Fichte y la "historia de la autoconciencia" de Schelling por una "historia de la formación de la conciencia". En ello coindicen los dos títulos que ha tenido la obra, primero el de "Ciencia de la experiencia de la conciencia" (cuando la obra se presentaba como la primera parte del sistema) y después el de "Ciencia de la Fenomenología del Espíritu", puesto que "historia de la experiencia y fenomenología son más bien dos aspectos de la misma cosa que dos métodos que caracterizan las distintas partes de la obra"24.

Hacemos esta breve alusión a este contexto para resaltar más lo propio del proyecto fenomenológico de Hegel, que consiste en la transformación del a priori kantiano, el a priori de todo conocimiento y toda acción y en cuyo conjunto se integra, todo nuevo conocimiento, transformación por la que el a priori queda transmutado en una historia de formación de la conciencia que cada individuo y cada época tiene a sus espaldas y que por tanto, siendo histórico, actúa como a priori. En resumen,

Erläuterung des Idealismus der Wissenschaftslebre, en: Schelling, Scbriften, WBG, Darmstadt, 1975, vol. 1, pp. 223-332, ref. p. 262.

${ }^{24}$ Siep, Ludwig, Der Weg der Pbänomenologie des Geistes, Suhrkamp, Frankfurt a.M., 2000, p. 63. 
se trata de una historización del transcendental kantiano: las condiciones a priori de posibilidad se convierten en realidad histórica que posibilita ${ }^{25}$.

Dado que el lugar central que ocupa la cuestión de la experiencia en la Fenomenología del espiritu de 1807, vamos a referirnos exclusivamente a ella, puesto que es sin duda el texto donde de manera más explícita y clara se plantea la cuestión ${ }^{26}$. En efecto, en esta obra cuida muy bien Hegel de exponer la certeza sensible o la percepción (y así sucesivamente los demás capítulos) como la conciencia sentiente o percipiente ( $y$ así sucesivamente todas las figuras de la conciencia o figuras de un mundo), de tal manera que la experiencia se convierte en algo formador y transformador de la conciencia: en la medida en que la conciencia hace experiencias, se va formando y transformando, tomando nuevas figuras. $\mathrm{Y}$ a la inversa, en la medida que el objeto va siendo más conocido se va desplegando la realidad en toda su complejidad y diversidad (ética, moral, cognitiva, social, política, religiosa, etc.), gracias a lo cual la conciencia adquiere una nueva figura.

De esta manera pretende Hegel mostrar que la autoconciencia pertenece a la ciencia misma, y consiguientemente mostrar la unidad de ambas ${ }^{27}$. Así Hegel exige que la ciencia, es decir, que el en sí "tiene que exteriorizarse y convertirse en para sí mismo, lo que quiere decir, pura y simplemente, que él mismo tiene que poner la autonconciencia como una con él". ( $P h d G 26 / 20$ ). Contra el dogmatismo de la concepción que la verdad consiste en una frase, en algo inmediato, Hegel desarrolla el programa de una ciencia que une la

25 Como afirma Agamben, Infancia e bistoria, p. 40: " $\mathrm{La}$ experiencia deja de ser simplemente un modo o un instrumento o un límite de la conciencia y se vuelve la esencia misma del nuevo sujeto absoluto: es su estructura de proceso dialéctico, de movimiento".

${ }_{26}$ De entre los numerosos estudios me permito citar Álvarez Gómez, Mariano, Experiencia y sistema. Introducción al pensamiento de Hegel, Univ. Pont., Salamanca, 1978; Heidegger, Martin, "Hegels Begriff der Erfahrung", en: Id., Holzwege, Klostermann, Frankfurt a.M., 41963, pp. 105-192; vers. cast.: "El concepto de experiencia de Hegel", en: Id., Caminos de bosque. Vers. cast. de Helena Cortés y Arturo Leyte, Alianza, Madrid, 1995, pp. 110-189.

27 Cfr. Weisser-Lohmann, Elisabeht, "Gestalten nicht des Bewusstseins, sondern einer Welt. Überlegungen zum Geist-Kapitel der Phänomenologie des Geistes", en: Köhler, Dietmar / Pöggeler, Otto (ed.), Hegel: Phänomenologie des Geistes, Akademie, Berlin, 2006, pp. 185-209, ref. pp. 185-191. 
exposición del saber absoluto con el principio de la realidad, la autoconciencia inmediata. Para ello se ha de mostrar el camino de la ciencia, por una parte, como el camino de la autoconciencia hacia el verdadero saber, y, por otra, este proceso debe ser una exteriorización del verdadero saber en el mundo. Para la autoconciencia este programa se presenta como una escalera hacia el verdadero saber. Ambas perspectivas se encuentran entretejidas en la exposición de la Fenomenología, por lo que es denominada tanto "exposición del saber que deviene", que se despliega (Darstellung des werdenden Wissens), como también "Ciencia de la experiencia de la conciencia". Ambas pespectivas se entrecruzan y se unen precisamente en el concepto de experiencia, de ahí el papel decisivo de este concepto, que viene dado por la función que Hegel asigna a la autoconciencia, pues la ciencia, en su exteriorización, se ve remitida a las experiencias de la autoconciencia, pues sólo en ellas las determinaciones de la ciencia son verdaderamente reales, solamente en ellas adviene "espíritu" a dichas determinaciones. Con ello ya resulta claro que en estas experiencias no se trata de experiencias sensoriales, de las que pudiera derivarse conocimientos. Experiencia incluye aquí todo lo que afecta al hombre: opiniones científicas, cosas de la vida, cuestiones de conciencia moral, experiencias religiosas, posiciones filosóficas. La pretension de Hegel es que la exposición fenomenológica deduzca y elabore esta conexión de saber y experiencia.

\subsection{Crítica al planteamiento kantiano}

De entrada, como casi siempre, puede afirmarse que Hegel asume el planteamiento kantiano. En concreto, es clara la afirmación de la experiencia como conocimiento. En este sentido debe entenderse esta lapidaria sentencia, según la cual "nada es sabido que no esté en la experiencia" (PhdG 558/468); el ámbito del conocimiento, incluso del saber, es idéntico con el de la experiencia.

Ahora bien, a partir de este punto básico en común, y siendo coherente con él, Hegel lanza una crítica radical al planteamiento kantiano, negando la distinción entre fenómeno y noúmeno. Si la experiencia es ya conocimiento, carecemos de cualquier punto de referencia para establecer dicha distinción, puesto que el en-sí no puede ser más que su ser para-nosotros (PbdG 71/57), el fenómeno es el ser-conocido, el manifestarse de lo absolutamente verdadero (PhdG 39/32), o, como Hegel argumenta de manera irónica, si del resultado, a saber del fenómeno, descontamos la parte que corresponde al sujeto, a fin de deducir el concepto de la cosa en sí, no nos vamos a encontrar con dicho 
concepto, sino más bien con la nada, es decir, nos encontramos en el momento anterior al proceso de conocimiento $(P b d G 64 / 51)^{28}$.

A partir de la negación de esta distinción y la consecuente identificación entre fenómeno y cosa en sí, se comprende también la superación de la distinción entre sujeto transcendental y sujeto empírico, por lo menos en el sentido de un conjunto de estructuras anteriores e independientes de toda experiencia, a la vez que forman el conjunto de condiciones para toda experiencia. Este supuesto sujeto que hace posible toda experiencia es a su vez fruto de todo el conjunto de experiencias que configuran la historia del espíritu humano, la historia de la humanidad. Y en este sentido es la experiencia misma la que hace posible nuevas experiencias; la nueva experiencia se hace sobre la base de la experiencia anterior que ha ido configurando y estructurando al sujeto en general, al cual el sujeto individual se va conformando mediante su propia formación, siendo ésta la escalera que le permite ponerse a la altura alcanzada por el espíritu.

\subsection{La experiencia como configuradora de la conciencia}

Kant, con su concepto de 'cosa en sí, daba a la cosa una existencia independiente del pensar e inaccesible. Para Hegel, en cambio, esta dualidad entre fenómeno y noúmeno, no tiene sentido ya que el fenómeno no es más que la aparición del noúmeno. El "en sí" se convierte en el "para la conciencia" o para nosotros y solamente podemos hablar de él en la medida en que tengamos noticia de él, que nos aparezca, que se convierta en objeto de nuestra conciencia, que lo podamos percibir, pensar.

"Cuando lo que primeramente aparecía como el objeto desciende en la conciencia a un saber de él y cuando el en sí deviene un ser del en sí para la conciencia, tenemos un nuevo objeto por medio del cual surge también una nueva figura de la conciencia, para la cual la esencia es ahora algo distinto de lo que era antes". (PhdG 74/59).

De esta manera surge un doble movimiento: por una parte, el conocer viene a producir siempre nuevos objetos en el saber, conociendo ampliamos nuestro mundo; $y$, por otra parte, junto con este crecimiento en

${ }^{28}$ Aunque según Siep no es claro que aquí se aluda a Kant, a su parecer más bien se apunta a Jacobi. Cf. Siep, Der Weg der Pbänomenologie des Geistes, p. 74. 
el saber se producen nuevas figuras de conciencia, no sólo nuevas tomas de conciencia, sino también nuevas actitudes, nuevas conciencias. Este doble movimiento que resulta de parte del sujeto y de parte del objeto es lo que finalmente entiende Hegel por experiencia:

"Este movimiento dialéctico que la conciencia lleva a cabo en sí misma, tanto en su saber como en su objeto, en cuanto brota ante ella el nuevo objeto verdadero, es propiamente lo que se llamará experiencia". (PhdG 73/58).

Experiencia es, por tanto, la sustitución de una falsa o incompleta o imperfecta convicción por otra verdadera más completa y perfecta; es lo que en términos religiosos se llama conversión, y en términos pedagógicoilustrados transformación de la conciencia ${ }^{29}$. Ahora bien, el modo de la sustitución viene definido con el término técnico: "dialéctico", que indica siempre un desarrollo y una superación de una contradicción, contiene la idea de una conservación y una elevación, pasando por una negación, puesto que los opuestos son negados en su independencia y llevados a una unidad superior. La experiencia es un movimiento dialéctico, porque en la experiencia se desarrolla algo ya dado, que es negado en su limitación y contradicción, y a la vez se conserva en un nivel ulterior, superior; es decir, que sobre la base de la experiencia tenida y superando sus limitaciones y contradicciones se llega a nuevas y más lúcidas experiencias. El sentido propio de experiencia en la Fenomenología es pues el de conversión, transformación de la conciencia ${ }^{30}$. La experiencia es el proceso por el que la conciencia pasa de una figura a otra, se transforma ${ }^{31}$.

Con ello Hegel da cuenta de la apertura característica de la experiencia: es movimiento generador de objetos y de nuevas figuras de conciencia. En este sentido no hay duda que Hegel sí ha prestado atención a qué pasa en la experiencia y no sólo a las condiciones de su posibilidad: qué pasa en el sujeto e incluso en el mundo del saber o de la ciencia. Pero, respecto a Kant, lo más destacado es que, según Hegel, en la experiencia se

${ }^{29}$ Siep, Der Weg der Phänomenologie des Geistes, pp. 63s.

30 Siep, Der Weg der Phänomenologie des Geistes, p. 77; Marx, Werner, Hegels Phänomenologie des Geistes. Die Bestimmung ihrer ldee in Vorrede' und 'EinIeitung", Klostermann, Frankfurt a.M., 1971, pp. 94-98.

${ }^{31}$ Marx, Hegels Phänomenologie, p. 90. 
forma el sujeto y se forma en la medida en que se abre al mundo, se despliega conociendo y actuando en el mundo. De esta manera las condiciones de posibilidad de la experiencia se van configurando por la experiencia, lo trascendental se historiza, se convierte en histórico.

\subsection{Límites}

A pesar de esta gran aportación al concepto de experiencia, cuenta también éste con su insuficiencia ${ }^{32}$. Intentemos especificar algunos aspectos.

En primer lugar, esta apertura característica de la experiencia no es una apertura indefinida, sino que tiene su tope: el sistema, el saber absoluto, su superación en ciencia, en lógica. Como en Aristóteles e incluso en el empirismo, también en Hegel la experiencia comporta su superación en saber, en ciencia. Es más, se puede afirmar que la experiencia se hace siempre desde una lógica.

En segundo lugar, por la misma razón que la experiencia se hace siempre con una lógica y bajo su imperio, la percepción misma es, en Hegel, no el encuentro con algo, con lo otro, sino que en último término es una experiencia de la conciencia misma, una autoexperiencia o experiencia de sí mismo. En este sentido puede observarse un rebajamiento en la valoración de la sensibilidad como apertura.

En tercer lugar, si, por una parte, la historia de la humanidad forma parte de la formación de la conciencia, y en este sentido se tiene en cuenta la historia y la situación histórica del sujeto, por otra parte, dicha historia queda estilizada o esquematizada como historia de la razón, de manera que el punto de partida no es el sujeto concreto, histórico, sino la idea de razón; el desarrollo de la conciencia sigue el "carril de una lógica previamente proyectada"33, de manera que la Fenomenologia "expone el sistema de las determinaciones conceptuales como historia. Pone las determinaciones especulativas como objetos de las figuras de la conciencia y así es una

${ }^{32}$ Kirchner, "Die Funktion existentieller Erfahrungen...", p. 168.

${ }^{33}$ Schmitz, Hermann, Hegels Logik, Bouvier, Bonn/Berlin, 1992, p. 301. 
exteriorización de la lógica desde la 'forma del concepto puro' en la historia de las experiencias, que la conciencia hace con sus objetos" 34 .

Finalmente, Hegel intenta representar los actos mentales sistematizadores de la razón como una actividad, en la cual, en último término, se capta y registra toda la realidad, y toda su abundancia de fenómenos y acontecimientos son ordenados en un sistema omnicomprensivo según leyes y procesos necesarios. La ciencia, que refleja en el pensamiento puro la estructura de toda la realidad comprensible racionalmente, es la lógica; de su sistema de categorías se deben derivar todos los fenómenos de la naturaleza y de la historia de la humanidad: "La Lógica se ha de entender, por tanto, como el sistema de la ciencia pura. Este reino es la verdad, tal como es en y para sí sin envolturas. Por ello se puede decir que este contenido es la exposición de Dios, tal como es en su propia esencia antes de la creación de la naturaleza y de un espíritu finito"35.

Con otras palabras, la ciencia de Hegel pretende anticipar incluso la creación del mundo y del hombre. Su sistema está de tal manera dispuesto que no puede ser puesto en cuestión por ninguna experiencia, sino que éstas solamente pueden tener la validez que les puede venir de su confirmación en el sistema. Tal como se desprende del texto citado, la estructura racional de la realidad está ya fijada, antes de que el espíritu finito, un ser humano racional, intente conocerla por medio de la experiencia y reflexionando sobre su experiencia.

\section{La experiencia es la existencia}

En el mismo concepto moderno de experiencia se puede observar una radicalización en el sentido de que este concepto va adquiriendo una densidad y una amplitud que lo convierte en un concepto

34 Pöggeler, Otto, Hegels Idee einer Phänomenologie des Geistes, Alber, Freiburg/München, 1973, p. 354. Sobre esta problemática cfr. Fulda, Hans Friedrich, Das Problem einer Einleitung in Hegels Wissenschaft der Logik, Klostermann, Frankfurt a.M., 1965, 21976; Düsing, Klaus, Das Problem der Subjektivität in Hegels Logik. Systematische und entwicklungsgeschichtliche Untersuchungen zum Prinzip des ldealismus und der Dialektik, Bouvier, Bonn, 1976 (HegeI-Studien Beiheft 15).

${ }^{35}$ Hegel, Wissenschaft der Logik. Hrsg. v. Georg Lasson, Meiner, Hamburgo, 1971, vol. 1, p. 31. 
omnicomprensivo, que va más allá del ámbito (de la teoría) del conocimiento, para adentrarse en lo constitutivo del sujeto y de la realidad. En efecto, en el mismo Hume, que, como se sabe en su planteamiento prescinde de la conciencia en sentido estricto: "la conciencia no es una realidad o cualidad del sujeto, antecedente a la puesta en marcha de los diversos dinamismos que calificamos como 'conscientes', sino que la conciencia es algo que deviene o se constituye en virtud de esos mismos dinamismos 'conscientes"', por este mismo hecho hay que afirmar que "no hay una conciencia distinta de la experiencia" 36 . La misma negación de una conciencia que sea en sí, anterior e independiente de la experiencia, parece que de alguna manera obliga a que se la considere como constituida por la experiencia, en sentido análogo a como Hume define al yo como un haz de percepciones ${ }^{37}$. En cambio, esta concepción no permite plantear la cuestión de si esta conciencia puede ser pensada como el conjunto de condiciones de posibilidad de adquisición de experiencia, aunque se insinúe, por ejemplo, en el hecho de considerar la costumbre como lo que hace unir cosas y hechos de una manera (por ejemplo la causalidad) que por sí mismos no permitirían.

En Kant se puede observar esta radicalización, por una parte, en su tendencia a identificar el mundo o la naturaleza, como compendio de los fenómenos (Inbegriff der Erscheinungen), con el campo de la experiencia ${ }^{38}$, y, por otra, en el hecho de poner como principio supremo del conocimiento y de la experiencia lo que integra el nuevo conocimiento y la nueva experiencia dentro del conjunto del conocimiento ya estructurado y configurado. Ciertamente que Kant mantiene la distinción entre trascendental y empírico, de manera que no puede afirmarse que el principio supremo de la condición de posibilidad de la experiencia sea la experiencia ya hecha de manera que la nueva sea integrable en ella, o que la hecha sea la condición de posibilidad de la nueva; pero sí que la actividad que lleva a

${ }^{36}$ Rábade, Sergio, 'La noción de experiencia en el empirismo inglés: Hume”, en: Id., Obras, Trotta, Madrid, 2004, vol. II, pp. 57-74, cita p. 59.

37 Hume, David., Tratado de la naturaleza bumana. 2 vols. Edic. de Félix Duque, Editora Nacional, Madrid, 1977 (Libro I, Parte IV, sección VI) vol. I, p. 400 . Aunque ésta sea la metáfora más conocida, Hume expone otras como son las del teatro y la república, que resultan mucho más cercanas a las teorías actuales de la identidad.

${ }^{38}$ Cfr. Holzhey, Kants Erfabrungsbegriff, pp. 248ss. 
cabo el sujeto o la conciencia trascendental parte de la experiencia hecha para integrar en ella la nueva.

En Hegel la radicalización apunta especialmente en el hecho de anular la distinción entre fenómeno y cosa en sí y entre conciencia empírica y transcendental, puesto que ésta última, en cuanto conjunto de las condiciones de posibilidad de la experiencia, está configurada por la experiencia misma, por la "historia de la formación de la conciencia". En Hegel mismo podemos ver anunciado -y por lo menos en parte realizadoel movimiento posterior que -a los ojos de Foucault- irá tomando la filosofía, por lo menos en la línea de la Aufklärung, por la que la filosofía se convierte en "la figura determinante de una época", considerándose a la vez a la época como "la forma de cumplimiento de esta filosofía", de manera que "la época aparecería como no siendo otra cosa que la emergencia y la manifestación, en sus rasgos fundamentales, de lo que era en su esencia la filosofía"39.

A partir de este esbozo de la configuración del concepto de experiencia en este período se pueden establecer -prescindiendo de otras no menos importantes, como el positivismo y el neopositivismo en sus diversas versiones, así como también el pragmatismo en sus distintas variantes- dos líneas de evolución: por una parte, partiendo del neokantismo, especialmente de Hermann Cohen, cuya estela será seguida por Fr. Rosenzweig y W. Benjamin; y, por otra, la fenomenología y la hermenéutica. En términos generales cabe afirmar que ambas líneas toman a Kant como punto de partida y se configuran tomando motivos del desarrollo crítico que Hegel hiciera del criticismo.

Así el neokantiano ${ }^{40} \mathrm{H}$. Cohen se sentía ligado especialmente con el principio según el cual sólo puede ser lo que es también pensable, siguiendo el

39 Foucault, Michel, “La vie: l'expérience et la science”, en: Id., Dits et éscrits 19541988. Vol. IV: 1980-1988. Édition établie sous la direction de Daniel Defert et François Ewald avec la collaboration de Jacques Lagrange, Gallimard, Paris, 1994, pp. 763-776, cita p. 765s.

${ }^{40}$ Kirchner, "Die Funktion existentieller Erfahrungen...”, pp. 167s. Cfr. Richardson, Alan W., "Conceiving, Experiencing, and Conceiving Experiencing: NeoKantianism and the History of the Concept of Experience", en: Topoi 22 (2003) 55267. 
principio de Parménides (B 5: "pues pensar [el ente] y ser es lo mismo"). Este principio ha marcado toda la historia de la filosofía y se encuentra también en la base del sistema hegeliano. En la equiparación de ente y pensable Cohen vio la posibilidad de demostrar, con ayuda de consideraciones matemáticas, la total ausencia de presupuestos del pensar sistemático en filosofía. Así en su obra Logik der reinen Erkenntnis (1914²) escribe: "Nosotros empezamos con el pensar. El pensar no debe tener ningún origen fuera de sí mismo. [...] El puro pensar debe llevar en sí mismo y exclusivamente a la generación de los conocimientos puros [...]. Se debe pues permanecer en la relación que Parménides forjó como identidad de pensar y ser. El ser es el ser del pensar. Por tanto el pensar es, en tanto que pensar del Ser, pensar del conocimiento"41. Desde esta interpretación platonizante del kantismo se puede detectar, de manera curiosa, como la misma afirmación de la identidad de pensar y ser conduce a la afirmación de que no hay nada absolutamente previo al conocer, a la experiencia. En esta misma línea se puede entender la importancia que W. Benjamin concede a la experiencia, como lo que realmente forma el sujeto, o cuya pérdida significa a la vez la aniquilación del individuo y su masificación. De ahí su afirmación, que viene a identificar la experiencia y la existencia: "Ta concreta totalidad de la experiencia, es decir, la existencia" 42.

La fenomenología ha destacado el concepto de experiencia ${ }^{43}$ también en una doble línea. Por una parte, por su atención al mundo de la vida, como el mundo de la conciencia prerreflexiva y como base de toda

${ }^{41}$ Hermann Cohen, Logik der reinen Erkenntnis in: Werke, hrsg. v. Helmut Holzhey. vol. VI, pp. 13. 15.

42 Benjamin, Walter, "Über das Programm der kommenden Philosophie" (1918), en: Gesammelte Schriften, vol. II/1, p. 171.

${ }^{43}$ En general sobre el concepto de experiencia en la fenomenología cfr. Landgrebe, Ludwig, "The Phenomenological Concept of Experience", en: Pbilosopby and Phenomenological Research 34 (1973: 1) 1-13. Sobre M. Merleau-Ponty cf. Pochuelo, Alicia G., "El origen de la experiencia humana", en: Pensamiento 53 (1997) 113-125. Sobre la experiencia en la filosofía existencialista cfr. Pieper, Annemarie, "Erfahrung in der Existenzphilosophie", en: Freudiger, Jürg/Graeser, Andreas/Petrus, Klaus (Hg.), Der Begriff der Erfabrung in der Pbilosopbie des 20. Jabrbunderts, Beck, München, 1996, pp. 153-177. 
reflexión ${ }^{44}$; y, por otra, poniendo en lo vivido (vécu) "el sentido originario de todo acto de conocimiento" 45 , de tal manera que en él se concentra no sólo el conocimiento sino la existencia misma ${ }^{46}$. Estas dos líneas convergen en el concepto gadameriano de experiencia como apertura a nuevas experiencias ${ }^{47}$. Seguramente se puede considerar como la última navegación en las aguas de la identificación entre experiencia y existencia la teoría de la identidad narrativa de Paul Ricoeur (1913-2005) ${ }^{48}$, en el sentido de que toma en consideración la quiebra del cogito cartesiano, y por tanto la disolución de toda

${ }^{44}$ Cfr. Welter, Rüdiger, Der Begriff der Lebenswelt. Theorien vortheoretischer Erfabrungswelt, Fink, München,1986.

${ }^{45}$ Foucault, 'La vie: l'expérience et la science", p. 773.

46 Hernández Borque, Francisca, "Hume y Merleau-Ponty, filósofos de la experiencia", en: Anales del Seminario de Metafísica 11 (1976) 63-104, traza paralelismos -semejanzas y divergencias- entre ambos planteamientos. De ahí el "duplicado" (empírico-transcendental) que Foucault cree detectar en todo discurso sobre el hombre, afectando tanto a la teoría del conocimiento como a la teoría de la existencia, y sin duda, también a la mayoría de los conceptos de experiencia. Cf. Foucault, Michel, Les mots et les choses. Une archéologie des sciences bumaines, Gallimard, Paris, 1974, pp. 329-333; vers. cast.: Las palabras y las cosas. Una arqueología de las ciencias bumanas, Siglo XXI, Madrid, 1997, pp. 310-313. Sobre esta crítica de Foucault al concepto de vécu cfr. Lawlor, L eonard, "Un écart intime (Part I): Foucault's critique of the concept of lived-experience (vécu)", en: Research in Pbenomenology 35 (2005) 11-28.

47 Gadamer, Hans-Georg, Wabrheit und Methode. Grundzüge einer philosophischen Hermeneutik, Tubinga, Mohr, (1960) 31972, pp. 329-344 (Vers. cast. de A. Agud Aparicio y R de Agapito: Verdad y método. Fundamentos de una bermenéutica filosófica, Sígueme, Salamanca, 1977, pp. 421-439).

48 Cfr. Ricoeur, Paul, "Existence et herméneutique", en: Id., Le conflit des interpretations, Seuil, París, 1969, pp. 7-28; Id., Temps et recit. I: L'bistoire et le recit, Paris: Seuil, 1983, vers. cast.: Tiempo y narración. 1: Configuración del tiempo en el relato bistórico, Cristiandad, Madrid, 1987, pp. 83ss., esp. "La trama: un modelo de concordancia" y "La discordancia incluida", pp. 94-106; Id., Temps et recit. III: Le temps raconté, Seuil, París, 1985, esp. pp. 352-359; Id., Soi-même comme un autre, Seuil, París 1990, pp. 137198, vers. cast.: Si mismo como otro. Traducción de Agustín Neira Calvo, Siglo XXI, Madrid, 1996; Id., "La identidad narrativa", en: Diálogo Filosófico núm. 24 (1992) 315324. Sobre esta cuestión cfr. Rubio Ferreres, José María, "Hermenéutica del sí mismo y narratividad. El problema de la identidad en Paul Ricoeur", en: Pedro Gómez García (coord.), Las ilusiones de la identidad. Cátedra, Madrid, 2000, pp. 253307. 
posible concepción sustancialista del mismo, pero sin renunciar al momento cartesiano, al momento reflexivo, sólo que éste no se da por la vía breve de la introspección, sino por el largo rodeo de la interpretación.

Para concluir he querido señalar algunas de las derivas que ha ido tomando el concepto de experiencia. En definitiva se trata de una profunda transformación que se gesta en el paso de Kant a Hegel, en el cual la experiencia pasa de ser tenida como el material (previo) del conocimiento, o su contenido, a significar la existencia misma o la identidad personal del sujeto.

\section{Resumen}

El concepto de experiencia, tanto en Kant como en Hegel así como en el proceso . de transformación que sufre con el paso de uno al otro, ofrece una gran complejidad. Aquí vamos a centrarnos en un solo aspecto, creo que nada marginal, a saber, la experiencia como constitutivo, como elemento o proceso constituyente del sujeto (tomado en su sentido más genérico), de forma que ella misma conforma el conjunto de condiciones de posibilidad para la apertura al mundo y la realización de nuevas experiencias, es más, es el conjunto que determina dicha apertura y la experiencia.

Con ello, en dicha transformación no sólo se produce la transformación del concepto moderno de experiencia, sino que se señala el campo en el que se va a debatir el concepto de experiencia en la filosofía contemporánea, aunque en este caso se traduzca en otros términos, tales como sí-mismo, subjetividad, existencia, identidad personal, identidad narrativa, etc.

Palabras clave: "Experiencia", "Kant”, “Hegel”, "Filosofía contemporánea".

\section{Abstract}

The concept of experience, in Kant as well as in Hegel, as well as the process of tansformation it undergoes in passing from one philosopher to the other, offers a great complexity. Here, we will take up in only one aspect, though not in the least marginal I believe; that is, experience as constituent, as an element or process constituent of the subject (taken in its most generic aspect), so that experience itself conforms the set of possibilility conditions for the opening up to the world and the realization of new experiences, and even more, the set which determines this opening and the experience. 
With it, in that transformation, not only the transformation of the modern concept of experience is produced, but also the field in which the concept of experience in contemporary philosophy is to be discussed is as well indicated, even though, in this case, it might be translated into other terms, such as self, subjectivity, existence, personal identity, narrative identity, etc.

Key words: "Experience”, “Kant”, “Hegel”, “Contemporary Philosophy”. 